\title{
Design, modeling, expression, and chemoselective PEGylation of a new nanosize cysteine analog of erythropoietin
}

This article was published in the following Dove Press journal:

International Journal of Nanomedicine

14 June 201 I

Number of times this article has been viewed

\author{
Reza Ahangari Cohan' \\ Armin Madadkar-Sobhani ${ }^{2,3}$ \\ Hossein Khanahmad' \\ Farzin Roohvand ${ }^{4}$ \\ Mohammad Reza Aghasadeghi ${ }^{4}$ \\ Mohammad Hossein Hedayati ${ }^{5}$ \\ Zahra Barghi ${ }^{5}$ \\ Mehdi Shafiee Ardestani ${ }^{4}$ \\ Davoud Nouri Inanlou' \\ Dariush Norouzian' \\ 'Research and Development \\ Department, Production and \\ Research Complex, Pasteur Institute \\ of Iran, Tehran, Iran; ${ }^{2}$ Department \\ of Bioinformatics, Institute of \\ Biochemistry and Biophysics, \\ University of Tehran, Tehran, Iran; \\ ${ }^{3}$ Department of Life Sciences, \\ Barcelona Supercomputing Center, \\ Barcelona, Spain; ${ }^{4}$ Hepatitis and AIDS \\ Department, Pasteur Institute of \\ Iran, Tehran, Iran; ${ }^{5}$ Quality Control \\ Department, Production and Research \\ Complex, Pasteur Institute of Iran, \\ Tehran, Iran
}

Background: Recombinant human erythropoietin (rhEPO) is considered to be one of the most pivotal pharmaceutical drugs in the market because of its clinical application in the treatment of anemia-associated disorders worldwide. However, like other therapeutic proteins, it does not have suitable pharmacokinetic properties for it to be administrated at least two to three times per week. Chemoselective cysteine PEGylation, employing molecular dynamics and graphics in in silico studies, can be considered to overcome such a problem.

Methods: A special kind of EPO analog was elicited based on a literature review, homology modeling, molecular dynamic simulation, and factors affecting the PEGylation reaction. Then, cDNA of the selected analog was generated by site-directed mutagenesis and subsequently cloned into the expression vector. The construct was transfected to Chinese hamster ovary/ $\mathrm{dhfr}^{-}$cells, and highly expressed clones were selected via methotrexate amplification. Ion-immobilized affinity and size exclusion (SE) chromatography techniques were used to purify the expressed analog. Thereafter, chemoselective PEGylation was performed and a nanosize PEGylated EPO was obtained through dialysis. The in vitro biologic assay and in vivo pharmacokinetic parameters were studied. Finally, E31C analog Fourier transform infrared, analytical SE-high-performance liquid chromatography, zeta potential, and size before and after PEGylation were characterized.

Results: The findings indicate that a novel nanosize EPO31-PEG has a five-fold longer terminal half-life in rats with similar biologic activity compared with unmodified rhEPO in proliferation cell assay. The results also show that EPO31-PEG size and charge versus unmodified protein was increased in a nanospectrum, and this may be one criterion of EPO biologic potency enhancement.

Discussion: This kind of novel engineered nanosize PEGylated EPO has remarkable advantages over rhEPO.

Keywords: nanoPEGylated EPO, cysteine PEGylation, pharmacokinetic property

\section{Introduction}

Erythropoietin (EPO) is synthesized in the kidney and plays an important role in the production and differentiation of erythroid progenitors to mature erythrocytes in hypoxia conditions, which is a process called erythropoiesis. ${ }^{1}$ A defect in the production of EPO in the body leads to a decrease in red blood cells (anemia), which results from chronic kidney disease, chemotherapy, and azidothymidine treatment of $\mathrm{HIV}^{2}$ The production of recombinant human EPO (rhEPO) is developed genetically by many biopharmaceutical industries, as it is widely used in anemia disorders. The available rhEPO is a highly glycosylated protein (40\% of molecular weight) and comprises 165 amino acids with a $\sim 35 \mathrm{kDa}$ molecular weight. ${ }^{3}$ Due to post-translational
Correspondence: Dariush Norouzian Pilot Biotechnology Department, Pasteur Institute of Iran,

Tehran, Iran

Tel +982I 66465406

Fax +9821 66402771

Email dnsa@pasteur.ac.ir 
modification, particularly in glycosylation pattern, and concerns about oncogenes in respect of human cell lines, it is expressed commercially in Chinese hamster ovary $(\mathrm{CHO})$ and baby hamster kidney cells. ${ }^{4,5}$

Many scientists have studied the expression of EPO in systems such as Escherichia coli ${ }^{6}$ Saccharomyces cervisiae, ${ }^{7}$ and bacullovirus, ${ }^{2}$ but because of rapid clearance from the circulation due to differences in sialic acid content, expressed EPOs did not have efficient in vivo activity. Nevertheless, CHO-expressed rhEPO has a half-life of 4-13 hours. In order for it to have therapeutic effect, it should be administered two to three times per week, which, in addition to the high cost of treatment, means frequent intravenous administration for patients. ${ }^{2}$ Therefore, there has been growing interest in the development of long-acting products to be administered less often than existing products.

One of the approved approaches for the improvement of pharmacokinetic properties of biologic products is PEGylation. PEGylation can lead to an increase in size, drug solubility, and bioavailability, while decreasing dose frequency as well as immunogenicity. ${ }^{8,9}$ The prominent marketing of some PEGylated proteins in the last decades has shifted the focus to the marketing of other PEGylated proteins. ${ }^{10}$

Basic problems with the existing PEGylated proteins are inefficient conjugation yield, the formation of heterogeneous mixtures, and a decrease in biologic activity as a result of nonspecific PEGylation. ${ }^{11}$ For example, the only marketed PEGylated form of rhEPO, CERA ${ }^{\circledR}$, was generated by conjugation of $30 \mathrm{kDa}$ methoxy-PEG via amide bonds to the N-terminal or the amino groups of lys-52 or lys-45. ${ }^{12,13}$ These drawbacks can be successfully compensated by chemoselective cysteine PEGylation, which is a strong tool for improvement of pharmacokinetic properties of therapeutic proteins. However, the addition of free cysteine residue to protein is not simple and occurs commonly in cysteine PEGylation because of the rare existence of free cysteine residue in the proteins. Introduced cysteine residue must not change the three-dimensional (3D) structure or stability of protein. ${ }^{14}$ In addition, factors affecting PEGylation must also be considered because experimental analysis of all stable cysteine analogs is a very time-consuming and costly process. Therefore, rational selection of suitable sites for replacement with cysteine residue is a critical step for this method. ${ }^{11,14}$ It has already been shown that an increase or decrease in the protein nanosize or charge by PEGylation may significantly affect protein biologic properties, including half-life, potency, and stability. ${ }^{15,16}$
In the present study, a novel nanosize PEGylated rhEPO has been developed and evaluated based on a literature review, homology modeling, molecular dynamic (MD) simulations, factors affecting PEGylation, and biologic assays that have a bigger nanosize, more negative charge, and better pharmacokinetic properties with the same in vitro biologic potency compared with intact rhEPO.

\section{Materials and methods Selection of a position for replacement by cysteine residue using an in silico approach}

A literature review was performed for finding nonstructural and nonfunctional residues. 3D structures of cysteine analogs were generated by homology modeling using MODELLER version 9.5 and a crystal structure of EPO (PDB code: 1EER, chain A, $1.9 \AA$ ) as a template (http://www.salilab.org). ${ }^{17}$ For each cysteine analog or native EPO, 10,000 molecules were generated, and the molecule corresponding to the lowest value of probability density function was selected. The quality of models was checked using discrete optimized protein energy (DOPE) score profile ${ }^{18}$ and PROCHECK (http://www. biochem.ucl.ac.uk). ${ }^{11}$ The stability of modeled analogs was examined by MD simulation using a GROMACS 3.3 package (http://www.gromacs.org) and Gromos96 force field. ${ }^{19} \mathrm{MD}$ simulations were carried out at a constant temperature $(300 \mathrm{~K})$ and pressure ( 1 bar) for 5-15 ns. After calculating the average structures corresponding to the last 500-2000 ps of MD simulations, modeled cysteine analogs with a root mean square deviation (RMSD) of less than $4 \AA$ from the initial structure were chosen for further analysis. Stable cysteine analogs were subjected to screening based on the RMSD from native EPO, surface area accessibility of engineered cysteine residues (cysteine-SAA), and pKa values of the thiol group. All-atom RMSDs between native EPO and the selected analogs were calculated by Qmol software (http://www.mbg.cornell.edu). ${ }^{20}$ The $\mathrm{pKa}$ of thiol groups was determined by PROPKA 2.0 Web interface (http://propka.ki.ku.dk). ${ }^{21}$ Cysteine-SAA in the modeled analogs was computed by GETAREA server (http:// curie.utmb.edu). ${ }^{22}$ Cysteine analogs with SSA more than 50\% were considered to be solvent exposed. Finally, one cysteine analog was selected for experimental analysis.

\section{Construction of an expression vector containing an engineered cysteine analog}

The cDNA of EPO was obtained by the reverse transcriptasepolymerase chain reaction (RT-PCR) method from Hep G2 
Cell line (ATCC No. HB-8065, USA) and cloned into the pTZ57R/T using first cDNA synthesis and a T/A cloning kit, respectively (Fermentas, Vilnius, Lithuania). A Histag and kozak sequence were added to the $\mathrm{C}$-terminal and N-terminal of EPO's cDNA with a forward primer, 5'-ATT ATG GGG GTG CAC GAA TGT CCT G-3', and a reverse primer, 5'-TCA ATG ATG ATG ATG ATG ATG GTC CCC TGT CCT GCA G-3', respectively. Replacement of glutamic acid at position 31 with cysteine was performed by the sitedirected mutagenesis method using a forward primer 31, 5'-GCT TGT CAC TGC AGC TTG AAT GAG AAT ATC AC-3', and a reverse primer 31, 5'-CAT TCA AGC TGC AGT GAC AAG CAC AGC CCG TCG TG-3'. All PCRs were performed with $P f u$ DNA polymerase to avoid unexpected mutations (Fermentas). The mutated gene was cloned into the optiCHO vector (Topo T/A cloning kit, Invitrogen Inc, Carlsbad, CA). Finally, the accuracy of the construction was confirmed by restriction analysis and sequencing.

\section{Expression of the engineered cysteine analog in the $\mathrm{CHO}$ cell line}

$\mathrm{CHO} / \mathrm{dhfr}^{-}\left(1.5 \times 10^{5}\right)$ cells (ATCC No. CRL-9096, USA) were cultured in Iscove's modified Dulbecco's medium supplemented with $10 \%$ (v/v) fetal bovine serum (GIBCO, Invitrogen Inc), penicillin (100 units/mL), streptomycin (100 $\mu \mathrm{g} / \mathrm{mL})$, hypoxanthine $(0.1 \mathrm{mM})$ (Sigma-Aldrich, Munich, Germany), thymidine (0.016 mM) (Sigma-Aldrich), and methotrexate $(0.002 \mathrm{mM})$ (Sigma-Aldrich) in a 24-well microplate. The final construct was linearized by $S s p$ I (Fermentas) and transfected using a lipofectamin $2000 \mathrm{kit}$ (GIBCO, Invitrogen, Inc). Transfected cells were selected in dialyzed $\alpha$-MEM medium containing $10 \%$ dialyzed FBS without any nucleosides (GIBCO, Invitrogen Inc). The resulting clones were subjected to gene amplification using increasing doses of methotrexate (Sigma-Aldrich). High-expressing clones were selected by EPO ELISA kit (Roche Diagnostics GmbH, Mannheim, Germany). RT-PCR and sequencing were carried out to confirm the existence of replaced cysteine codons at the mRNA level, first using a cDNA synthesis kit either by oligo-dT, random hexamer, or specific primers (Fermentas). Finally, secretion of cysteine analogs was analyzed by sodium dodecyl sulfate polyacrylamide gel electrophoresis (SDS-PAGE) in a $12 \%$ resolving gel at $200 \mathrm{~V}$ for $\sim 50$ minutes using the Mini-PROTEIN ${ }^{\circledR}$ Tetra Cell system (Bio-Rad Laboratories, Hercules, CA) and visualized by the Coomassie brilliant blue staining method. ${ }^{23}$ Electroblotting was performed using a Mini Trans-Blot ${ }^{\circledR}$ electrophoretic transfer cell (Bio-Rad
Laboratories) to Whatman nitrocellulose membrane (Schleicher and Schuell, Riviera Beach, FL) for 1 hour. Membrane was blocked in 5\% bovine serum albumin and $0.1 \%$ Tween 20 in phosphate buffered saline (PBS) at $4^{\circ} \mathrm{C}$ overnight. The identification of expressed E31C analog was performed by employing the mouse monoclonal antibody against rhEPO (GeneTex Inc, Irvine, CA) and goat antimouse immunoglobulin G (HRP) (GeneTex Inc) as a primary and secondary antibody for 1 hour at room temperature. The band was developed using a DAB chromogenic substrate (Sigma-Aldrich).

\section{Purification of the expressed cysteine analog}

Cell culture supernatant was concentrated 10-fold using a Labscale TFF system (Millipore, Billerica, MA) equipped with a PXC010C50 cassette (Pellicon XL, Millipore). Simultaneously, buffer exchange was carried out three times with PBS. An ion immobilized affinity chromatography (IMAC) was applied for purification of the cysteine analog using $5 \mathrm{~mL}$ Histrap columns (GE Healthcare Inc, Princeton, $\mathrm{NJ})$. The column was pre-equilibrated with $0.02 \mathrm{M}$ sodium phosphate $0.5 \mathrm{M} \mathrm{NaCl}$ and $20 \mathrm{mM}$ imidazole adjusted to $\mathrm{pH}$ 7.4. After sample loading and washing of columns with equilibration buffer, anchored cysteine analog was eluted with $0.02 \mathrm{M}$ sodium phosphate $0.5 \mathrm{M} \mathrm{NaCl}$ and $0.5 \mathrm{M}$ imidazole ( $\mathrm{pH}$ 7.4). An additional purification step was performed for elimination of imidazole and probable dimers by size exclusion chromatography (SEC) (HiLoad Superdex 75 prep grade, GE Healthcare). In this step, a solution containing $50 \mathrm{mM}$ sodium phosphate and $150 \mathrm{mM}$ sodium chloride $(\mathrm{pH} 7)$ was used as an equilibration and elution buffer. All fractions containing cysteine analog were pooled and adjusted to the desired concentration using Microcon ${ }^{\circledR}$ centrifugal filter devices (Millipore). Finally, purified analog was aliquoted and stored at $-80^{\circ} \mathrm{C}$ for further analysis.

\section{Chemoselective PEGylation of engineered cysteine analog}

PEGylation reaction was performed at room temperature and under nitrogen gas. Briefly, a 30-fold molar excess of TCEP (Tris [2-carboxyethyl] phosphine- $\mathrm{HCl}$ ) (Sigma-Aldrich) and a 30-fold molar excess of $30 \mathrm{kDa}$ mPEG-maleimide (SunBio Inc, Anyang City, South Korea) were added to a solution of E31C analog $(250 \mu \mathrm{g} / \mathrm{mL})$ in $50 \mathrm{mM}$ Tris buffer $\mathrm{pH} 8.0$ and incubated for 3 hours. Then, the PEGylation reaction was terminated by adding 10 -fold Tris buffer ( $20 \mathrm{mM}, \mathrm{pH} 8.0)$. Finally, the reaction was dialyzed against PBS, concentrated 
using Microcon ${ }^{\circledR}$ centrifugal filter devices (Millipore), and stored at $-80^{\circ} \mathrm{C}$ for further analysis.

\section{Characterization, size, and zeta potential of PEGylated and unmodified cysteine analog}

SE-high-performance liquid chromatography (HPLC) (Shodex PROTEIN KW-802.5 SEC column, Shodex, Japan) was employed for analysis of the protein modification using $50 \mathrm{mM}$ sodium phosphate containing $150 \mathrm{mM}$ sodium chloride ( $\mathrm{pH} 7$ ) as an equilibration and elution buffer. $20 \mu \mathrm{L}$ of each sample was injected separately and monitored at $214 \mathrm{~nm}$. Also, the spectral changes of the E31C analog were analyzed by Fourier transform infrared (FTIR) spectroscopy (Thermo-Nicolet, NEXUS 870, Waltham, MA) before and after the conjugation. Through changes in IR spectra, we were able to show the surface conjugation and clarify which group was involved in the conjugation process. In addition, EPO31-PEG and E31C analog $(0.02 \mathrm{mg} / \mathrm{mL})$ were checked for any changes in the size and zeta potential distribution by DLS technique (Malvern, Zetasizer Nano ZS, Worcestershire, UK).

\section{Biological assays and pharmacokinetic studies}

In vitro assays of all samples were carried out based on proliferation of UT-7 cells as follows. UT-7 cells (ACC 137; DMSZ, Braunschweig, Germany) were grown in $\alpha$-MEM supplemented with $20 \% \mathrm{FBS}, 40 \mathrm{mg} / \mathrm{mL}$ gentamycin, $2 \mathrm{mM}$ glutamine, and $5 \mathrm{ng} / \mathrm{mL}$ GM-CSF (Sigma-Aldrich). Five thousands cells were seeded in each well of a 96-plate. Twofold serial dilutions of the protein samples and standard were prepared in assay media. One hundred $\mu \mathrm{L}$ of the diluted protein samples was added in triplicate to each well of a 96-well microplate. Ten thousand cells were washed and seeded in each well and incubated at $37^{\circ} \mathrm{C}$ in humidified $5 \% \mathrm{CO}_{2}$ for 3 days. PBS and $200 \mathrm{ng} / \mathrm{mL}$ rhEPO (R\&D Systems, Minneapolis, $\mathrm{MN}$ ) were added to the cultured cells as a negative and positive control, respectively. Proliferation of cells was measured by MTT assay, a technique based on the reduction of MTT to formazan by viable cells. ${ }^{24}$ Finally dose-response curves were drawn for rhEPO, E31C analog, and EPO31PEG. Animal experiments were performed with the approval of the Animal Ethics Committee of the Pasteur Institute of Iran. The five Sprague-Dawley rats $(300 \mathrm{~g} \pm 30 \mathrm{~g}$ weight $)$ in each group received a single intravenous dose of $30 \mu \mathrm{g}$ protein $/ \mathrm{kg} .{ }^{12}$ Blood samples were collected from the lateral tail vein at 15 minutes, 1 hour, 8 hours, 24 hours, 48 hours,
96 hours, 120 hours, and 144 hours after the injection. The collected samples were centrifuged at $1500 \mathrm{~g}$ for 12 minutes, and separated plasma was analyzed using an EPO ELISA kit (Roche Diagnostics GmbH). The terminal half-life and the area under the curve were determined using linear regression of the last three plasma concentrations and a linear trapezoidal method with extrapolation to infinity, respectively. Protein concentrations were determined using a Bradford dye binding assay. Statistical analysis of the data was performed using Student's $t$-test and one-way multivariate analysis of variance to calculate the level of significance value ( $P$-value). Values of $P<0.05$ were considered statistically significant.

\section{Results}

\section{Selection of a position for replacement with cysteine residue using an in silico approach}

From 165 residues in the EPO, 115 positions were excluded based on a literature review (Table 1). The 3D structures of selected analogs were generated successfully from related sequences using homology modeling because of high homology (above 95\%) between the template and cysteine analogs. The quality of modeled analogs was verified by Ramachandran plot and DOPE score profile. Over $98 \%$ of residues in the generated models are located in regions based on $\psi$ and $\varphi$ angels, as shown in Figure 1A for E31C analog. DOPE score profile of cysteine analogs did not show any energy differences compared with the template, as depicted in Figure 1B for E31C analog. MD analysis was performed for all analogs in 5-15 ns, and RMSD versus time was plotted for the evaluation of model stability and average structure calculations (Figure 1C). Stable cysteine analogs during MD simulations (40 analogs) were subjected to screening based on the SAA of replaced cysteine residue (Table 2), and 13 exposed cysteine analogs were selected. Then, $\mathrm{pKa}$ values

Table I Exclusion of residues involved in receptor binding or structural stability of erythropoietin, based on a literature review

\begin{tabular}{lll}
\hline Number of amino acids & Exclusion criteria & Reference \\
\hline $8-26,55-83,90-1 \mid 2,138-161$ & $\begin{array}{l}\text { Essential for } \\
\text { secretion }\end{array}$ & 28 \\
& $\begin{array}{l}\text { Conformational } \\
\text { change }\end{array}$ & 28 \\
$43-54$ & $\begin{array}{l}\text { Glycosylation site } \\
\text { Disulfide bond }\end{array}$ & $3,43,44$ \\
$38-40,24-26,83-85$ & $\begin{array}{l}\text { Receptor binding } \\
\text { site I }\end{array}$ & 32 \\
$29,33,7,161$ & Receptor binding & 32 \\
$20,44-47,13 \mid, 140,143,147,150$ & \\
$8,14,97,100,103,104$ & site 2 & \\
\hline
\end{tabular}


A

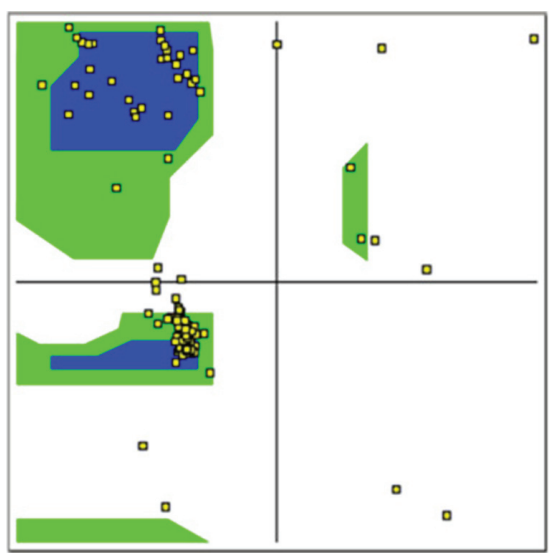

B

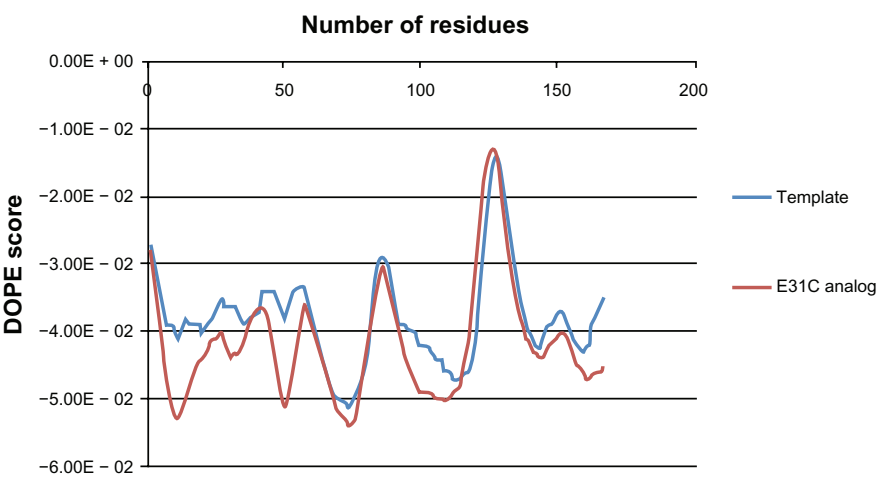

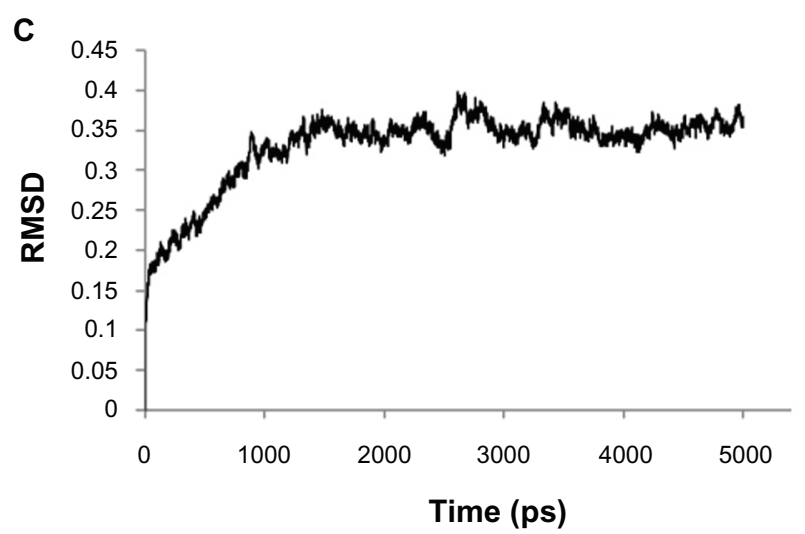

Figure I Quality assessment of the modeled three-dimensional structure of the R3IC analog as a sample. A) $>98 \%$ of residues are in the allowed regions of the Ramachandran plot. B) Discrete optimized protein energy (DOPE) score profile of modeled E3IC analog and template. C) Root mean square deviation (RMSD) plot for modeled E3 IC analog during molecular dynamic simulation. The fluctuations of the modeled structure reached plateau after $\sim 500$ ps of simulation.

of the thiol group and whole RMSD versus native EPO were determined (Table 3). Finally, based on having maximum cysteine-SSA and pKa first and then the minimum RMSD from native EPO in the final average structure, the E31C analog was chosen for experimental analysis.

\section{Construction, expression, and biologic evaluation of E3IC analog}

The natural cDNA of EPO was obtained by the RT-PCR method and T/A cloned into pTZ57R/T. The addition of His-tag, kozak sequence, and replacing of glutamic acid by cysteine codon were performed simultaneously by overlap extension PCR using respective primers (Figure 2). The mutated gene was cloned successfully into an optiCHO vector, and then the accuracy of the construct was confirmed by restriction analysis and sequencing (data not shown). The linearized construct was transfected into $\mathrm{CHO} / \mathrm{dhfr}^{-}$cells, and expressing clones were obtained in selective mediums. The E31C-expressing clones were subjected to methotrexate amplification (Figure 3A), and a high-expressing clone was selected using the ELISA method. The existence of replaced cysteine codon in the mRNA was confirmed by RT-PCR and sequencing (data not shown). The identification of expressed cysteine analog was carried out using an anti-EPO antibody on nitrocellulose membrane (Figure 3C). The verified clone was cultured, and harvested supernatants were concentrated and immediately purified using IMAC and SE, as described in Materials and methods (Figure 3B). The biologic tests did not show any distinction in the activity of rhEPO, and the $\mathrm{E} 31 \mathrm{C}$ analog $\mathrm{EC}_{50}$ (protein concentration achieving $50 \%$ of the maximum effect) for E31C analog and rhEPO were obtained, $0.53 \pm 0.03$ and $0.52 \pm 0.04$, respectively (Figure 4A).

\section{Characterization of PEGylated and unmodified analog}

The purified E31C analog and the PEGylated form were monitored by injection of each sample to an analytical SE-HPLC. As revealed in the chromatogram, the peak PEGylated form was eluted from the column $\sim 6$ minutes after the injection, which is earlier than the unmodified analog, as expected (Figure 5). The unmodified protein 
Table 2 Stability of modeled analogs after molecular dynamic simulations and surface area accessibility (SAA) of replaced cysteine

\begin{tabular}{|c|c|c|c|c|c|}
\hline $\begin{array}{l}\text { Cysteine } \\
\text { analog }\end{array}$ & Stability & $\begin{array}{l}\text { SAA } \\
\text { (\%) }\end{array}$ & $\begin{array}{l}\text { Cysteine } \\
\text { analog }\end{array}$ & Stability & $\begin{array}{l}\text { SAA } \\
\text { (\%) }\end{array}$ \\
\hline AIC & Stable & 48.8 & EIITC & Stable & 6.1 \\
\hline $\mathrm{P} 2 \mathrm{C}$ & Unstable & & AlI8C & Stable & 2.8 \\
\hline P3C & Stable & 6.7 & $1119 C$ & Stable & 47.4 \\
\hline R4C & Stable & 54.2 & SI20C & Unstable & \\
\hline L5C & Stable & 60.6 & $\mathrm{PI} 2 \mathrm{IC}$ & Stable & 17.6 \\
\hline $16 \mathrm{C}$ & Stable & 22.1 & $\mathrm{PI} 22 \mathrm{C}$ & Stable & 7.7 \\
\hline $\mathrm{T} 27 \mathrm{C}$ & Stable & 57.1 & DI23C & Stable & 88.7 \\
\hline G28C & Unstable & & $\mathrm{A} 124 \mathrm{C}$ & Stable & 0.7 \\
\hline $\mathrm{A} 30 \mathrm{C}$ & Unstable & & $\mathrm{A} 125 \mathrm{C}$ & Stable & 51.8 \\
\hline E3IC & Stable & 100.0 & $\mathrm{SI} 26 \mathrm{C}$ & Stable & 28.2 \\
\hline $\mathrm{H} 32 \mathrm{C}$ & Stable & 45.6 & AI27C & Stable & 30.1 \\
\hline S34C & Unstable & & $\mathrm{A} I 28 \mathrm{C}$ & Stable & 0.0 \\
\hline L35C & Stable & 0.0 & $\mathrm{PI} 29 \mathrm{C}$ & Stable & 3.7 \\
\hline N36C & Stable & 48.1 & LI30C & Stable & 78.1 \\
\hline E37C & Unstable & & $\mathrm{TI} 32 \mathrm{C}$ & Stable & 25.8 \\
\hline V4IC & Stable & 0.0 & $1133 C$ & Stable & 41.1 \\
\hline P42C & Stable & 10.3 & $\mathrm{TI} 34 \mathrm{C}$ & Stable & 52.2 \\
\hline Q86C & Unstable & & $\mathrm{A} 135 \mathrm{C}$ & Unstable & \\
\hline P87C & Unstable & 48.7 & DI36C & Unstable & \\
\hline W88C & Stable & 0.9 & TI37C & Stable & 43.7 \\
\hline E89C & Stable & 96.6 & $\mathrm{R} 162 \mathrm{C}$ & Stable & 72.9 \\
\hline GII3C & Stable & 9.4 & TI63C & Stable & 51.9 \\
\hline AlI 4 C & Stable & 0.0 & GI64C & Stable & 42.4 \\
\hline Q1I5C & Stable & 46.8 & DI65C & Stable & 9.0 \\
\hline $\mathrm{K} 116 \mathrm{C}$ & Stable & 72.6 & $\mathrm{R} 166 \mathrm{C}$ & Stable & 70.8 \\
\hline
\end{tabular}

peak appeared $\sim 20$ minutes after the injection. Surprisingly, biologic activity of EPO31-PEG indicated no significant difference either by rhEPO or by E31C analog (Figure 4A). Also, in animal experiments, illustrated in Figure 4B, EPO31PEG showed a five-fold terminal half-life compared with the $\mathrm{E} 31 \mathrm{C}$ analog. In the FTIR spectra of engineered analog, a peak appeared at $2414 \mathrm{~cm}^{-1}$ (Figure 6A), which endorsed the substitution of free cysteine, whereas it was not observed in the rhEPO spectrum (data not shown). After PEGylation of the engineered analog with $30 \mathrm{kDa}$ PEG-maleimide, a peak at $2414 \mathrm{~cm}^{-1}$ was missed and a peak raised at $2486 \mathrm{~cm}^{-1}$ (Figure 6B). This peak was related to $\mathrm{C}-\mathrm{H}$ bound conjugated
PEG polymer and confirmed that conjugation occurred in the free thiol group of replaced cysteine. The PEGylated analog showed an approximately two-fold increase in nanosize (from $3.1 \mathrm{~nm} \pm 0.3 \mathrm{~nm}$ to $5.9 \mathrm{~nm} \pm 0.2 \mathrm{~nm}$ ) relative to the unmodified protein (Figure 7A). Zeta potential results also showed an increase in negative charge of PEGylated analog due to attachment of the PEG polymer (Figure 7B). Finally, animal studies showed that PEGylated analogs have a lower elimination rate than unmodified analogs, as well as other improved pharmacokinetic parameters (see Table 4).

\section{Discussion}

Covalent attachment of PEG to proteins via cysteine residue, known as cysteine PEGylation, is considered an extremely efficient approach to circumventing product heterogeneity and loss of biologic activity characteristic of amine-PEGylation. ${ }^{25}$ Several grants were allocated to Bolder BioTechnology Inc (www.bolderbio.com) by the National Institutes of Health for cysteine PEGylation of therapeutic proteins. This method has some limitations in practice. Functional or structural stability of cysteine analogs and factors affecting conjugation must be considered, because experimental analysis of all positions in the protein is a very time-consuming and costly process. ${ }^{14}$

Desjarlais and Zalevsky ${ }^{26}$ reported a computational method for rational cysteine PEGylation. The method determines the ratio of the ranges of motion of the PEG for protein alone versus protein complexed with its receptor. This ratio is related to activity when PEG is coupled at a specific site in the protein, but this method does not evaluate the effects of introduced free cysteine on the conformation and stability of the protein. Therefore, to the authors' knowledge, there is no straightforward approach for rational site selection that comprises all aspects of cysteine PEGylation.

Our in silico approach consists of three steps. The first step is the selection of sites that do not involve protein function. This step can be carried out easily by a literature

Table 3 All-atom root mean square deviation (RMSD) and $\mathrm{pKa}$ values of the thiol group for stable cysteine analogs that had solvent-exposed cysteine

\begin{tabular}{llllllll}
\hline Analog & RMSD $(\mathbf{A})$ & pKa & SAA (\%) & Analog & RMSD (A) & pKa & SAA (\%) \\
\hline R4C & 2.991 & 9.00 & 54.2 & AI25C & 3.440 & 7.12 & 51.8 \\
L5C & 2.889 & 9.00 & 60.6 & LI30C & 3.739 & 9.00 & 78.1 \\
T27C & 3.250 & 8.03 & 57.1 & TI34C & 2.985 & 8.91 & 52.2 \\
E3IC & 2.942 & 9.00 & 100.0 & RI62C & 3.127 & 8.38 & 72.9 \\
E89C & 2.706 & 9.00 & 96.6 & TI63C & 3.204 & 8.64 & 51.9 \\
KII6C & 4.025 & 8.56 & 72.6 & RI66C & 3.116 & & 8.89 \\
DI23C & 3.628 & 9.00 & 88.7 & & & & 70.8 \\
\hline
\end{tabular}

Abbreviation: SAA, surface area accessibility. 


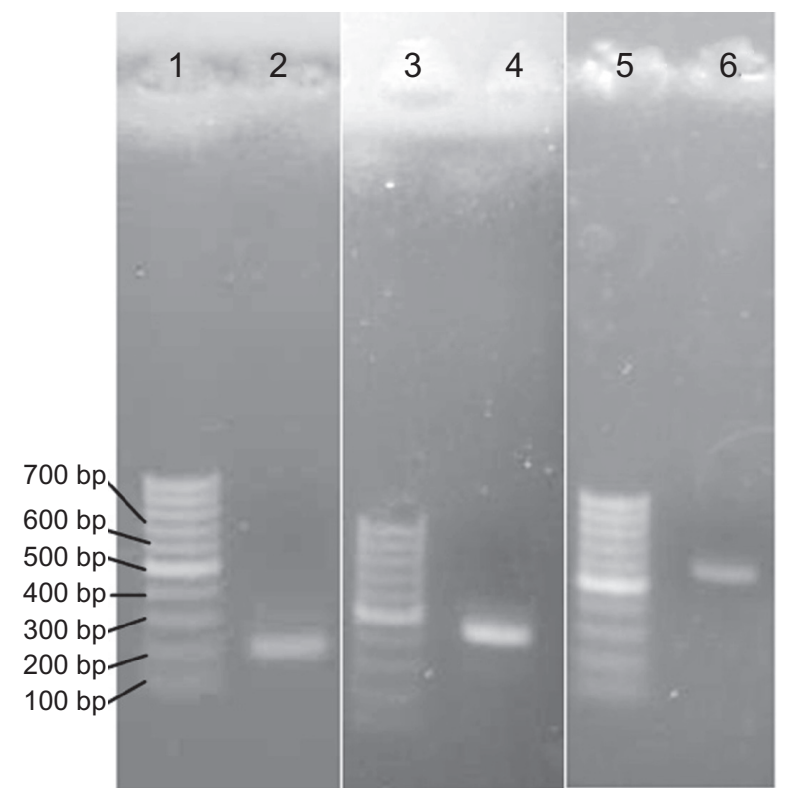

Figure 2 Replacement of glutamic acid codon with cysteine codon using sitedirected mutagenesis. Lanes I, 3, and 5: 100 bp markers (Fermentas, Lithuania); lane 2: first fragment of mutated cDNA containing kozak sequence and replaced cysteine codon ( $200 \mathrm{bp})$; lane 4 : second fragment of mutated cDNA containing His-tag and replaced cysteine codon ( $400 \mathrm{bp})$; and lane 6: the complete cDNA of E3IC analog ( $600 \mathrm{bp})$.

review, because almost all marketed recombinant proteins have been well studied. In this way, exclusion of residues involved in functional or structural stability of EPO was carried out successfully by literature review because of known EPO receptor binding sites (see Table 1).

In the second step, structural stability of analogs due to cysteine substitution must be evaluated. For this purpose, 3D structures of cysteine analogs must be subjected to MD simulation. ${ }^{27}$ As indicated in Table 3, the selected analog modeling and MD simulations revealed many possible sites
( $\sim 40$ positions) in the EPO structure for cysteine residue replacement, which did not disturb EPO structure by RMSD measurement. These findings are consistent with previous studies that examined bioactivity of mutated EPOs by sitedirected mutagenesis. ${ }^{2,28,29}$

In the third step, PEGylation factors must be assessed on stable modeled analogs. Factors affecting protein PEGylation include SAA, inherent nucleophilicity, and $\mathrm{pKa}$ of residue. Other factors, such as time, temperature, $\mathrm{pH}$, and protein/ PEG ratio, depend on the PEGylation method and must be determined empirically. ${ }^{30,31}$ In the present study, PEGylation factors related to the protein were analyzed on modeled stable analogs of EPO. The thiol group of introduced cysteine residue must be accessible for a successful attack on PEG molecules.$^{30}$ Therefore, cysteine analogs with low thiol SAA must be excluded. Also, PEG attacks on the cysteine residue on the protein surface are dependent on the nucleophilicity of cysteine residue. PEG attacks will take place easier when the $\mathrm{pH}$ of PEGylation reaction is higher than the $\mathrm{pKa}$ of the thiol group. Therefore, cysteine residues have $\mathrm{pKa}$ values less than the $\mathrm{pH}$ of the PEGylation reaction and are preferred for cysteine PEGylation. ${ }^{25}$ In combination with factors affecting PEGylation, only 13 positions remained for the further evaluations. As listed in Table 3, the selected positions were located structurally in the N-terminal, C-terminal, and AB, $\mathrm{BC}$, and $\mathrm{CD}$ loops of EPO that are not involved in stability or activity. Among the remaining analogs, the E31C and E89C analogs showed suitable criteria, including high SAA and low conformational change relative to the EPO.

Our in silico findings are in agreement with another study, by Desjarlais and Zalevsky, ${ }^{26}$ that measured the ratio of the ranges of PEG motion. It concluded that the best positions
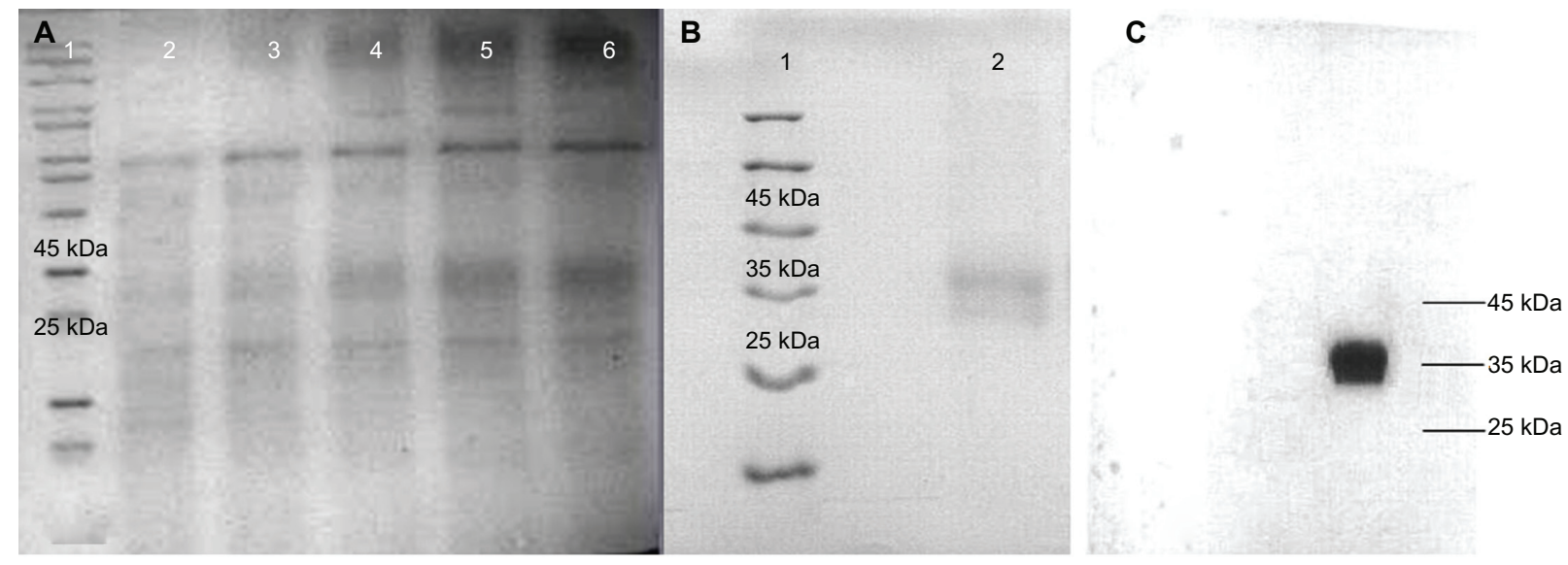

Figure 3 A) Methotrexate amplification of E3IC analog (lanes 2, 3, 4, 5, and 6), visualized by Coomassie brilliant blue staining method. B) Sodium dodecyl sulfate polyacrylamide gel electrophoresis analysis of purified E3IC analog ( $35 \mathrm{kDa})$ using immobilized affinity chromatography and size exclusion. C) Western blot analysis of E3IC analog using monoclonal mouse antierythropoietin antibody. 

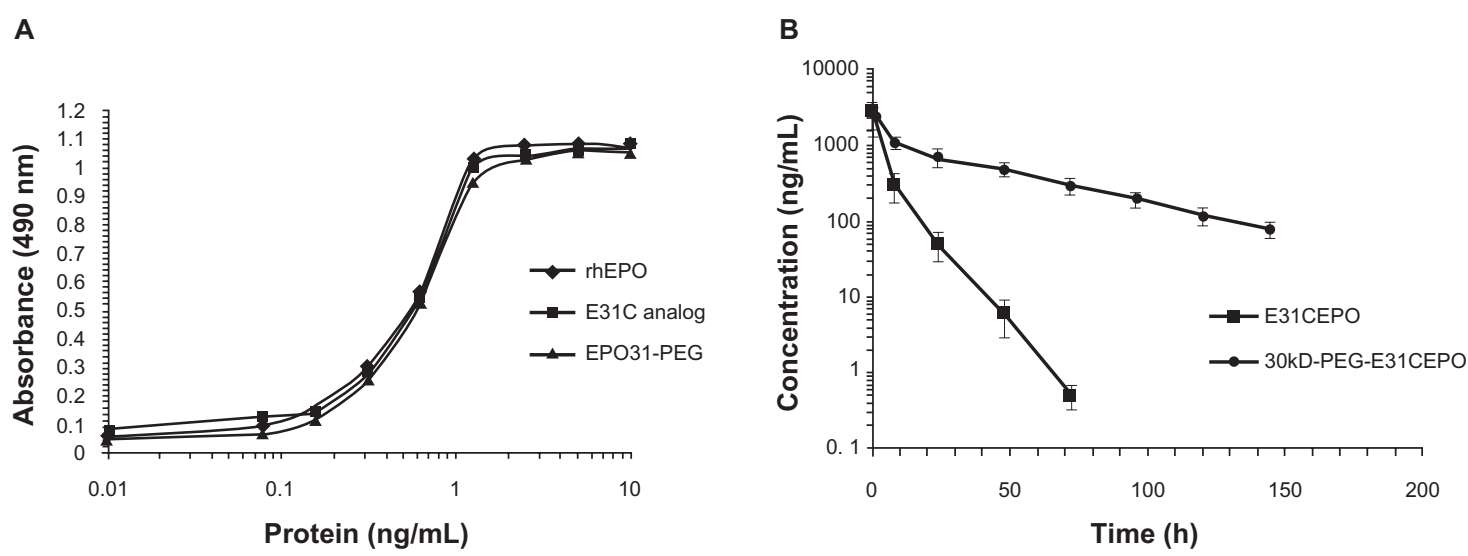

Figure 4 A) In vitro biologic assay based on proliferation of UT-7 cells. The EC 5 (protein concentration achieving 50\% of maximum effect) for E3IC analog and EPO3I-PEG were obtained $(0.53 \pm 0.03$ and $0.54 \pm 0.08$, respectively), similar to recombinant human erythropoietin (rhEPO) (0.52 \pm 0.04$)$. B) Single intravenous injection of EPO3IPEG showed five-fold terminal half-life for unmodified protein in rats ( 36.47 hours \pm 6.5 hours vs 7.22 hours \pm 1.3 hours). Protein concentrations in all experiments were determined using Bradford dye binding assay.

in the EPO for conjugation either by $2 \mathrm{kDa}$ or $10 \mathrm{kDa}$ PEG are Glu31, Ser85, and Ala125, which have optimal predicted conjugation efficiencies, stability, and functional activity. Substitution of Ser85 with cysteine disturbs N-glycosylation at Asn83, which is essential for in vivo activity of EPO. Long et $\mathrm{al}^{2}$ also showed that $\mathrm{S} 85 \mathrm{C}$ analog is glycosylated partially at position Asn83. The N85C analog was excluded in the first step of our approach. It must be noted that Desjarlais and Zalevsky ${ }^{26}$ performed their simulations on the 1EER, which does not contain any glycosyl chain, but Ser126 is glycosylated in expressed EPO. Therefore, spatial restriction of the glycosyl chain at position 126 may influence the coupling efficiency of the polymer negatively, but, as indicated in Table 3, the pKa value of the thiol group in Ala125 is lower than the other selected analogs, which may compensate for lower thiol accessibility. The $\mathrm{T} 27 \mathrm{C}$ analog has a similar condition as A125. The previously reported T26C analog lacks glycosylation at position 24 , which affects in vivo bioactivity due to a decrease in sialic acid content and

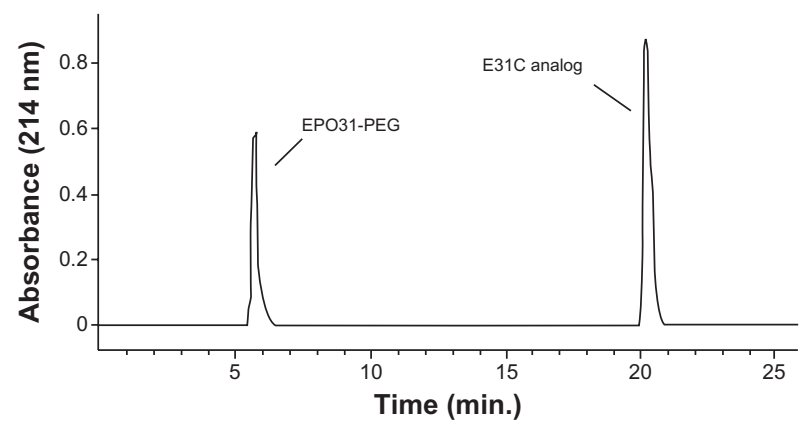

Figure 5 Size exclusion high-performance liquid chromatography chromatogram of PEGylated and unmodified analog. The peak at 6 minutes is related to EPO3I-PEG, and the second peak is attributed to E3IC analog eluted at 20 minutes after injection. leads to rapid clearance from circulation. ${ }^{2}$ Therefore, cysteine substitution at this position does not seem to be logical. Our data reveal that neighbor residue Thr27 is more suitable than Thr26 because cysteine substitution at this position does not affect $\mathrm{N}$-glycosylation at position 24 . As indicated in Table 3, the E89C analog has good criteria for cysteine PEGylation, high SAA and low RMSD, which is consistent with the previous study by Long et al. ${ }^{2}$ Even being PEGylated with a highmolecular-weight (20 kDa) PEG-maleimide, the E89C analog shows the same activity. ${ }^{2} \mathrm{~L} 130 \mathrm{C}$ and $\mathrm{T} 134 \mathrm{C}$ analogs are not suitable for PEGylation with a high-molecular-weight PEG because of their proximity to the R131, which is involved in receptor binding site 1 of EPO. PEGylation at these positions with $30 \mathrm{kDa}$ PEG can reduce receptor binding affinity of PEGylated forms. ${ }^{32}$ PEGylation of positions located in the N-terminal (R4 and L5) and C-terminal (R162, T163, and $\mathrm{R} 166)$ in the EPO are not logical because of spatial restriction in interaction between EPO and its receptors. As shown by Long et $\mathrm{al},{ }^{2}$ the attachment of $20 \mathrm{kDa}$ PEG at positions A1, $\mathrm{P} 3$, and T163 decreases receptor binding affinity. In addition, it has been reported that terminal arginine of EPO is deleted from the CHO-expressed rhEPO. Therefore, remaining replaced cysteine at this position must be proved. ${ }^{33} \mathrm{~K} 116 \mathrm{C}$ and D123C analogs show higher RMSD than other analogs, but both analogs have reasonable cysteine accessibility with closer $\mathrm{pKa}$ value. As reported, PEGylation at neighbor position Q115 with a $20 \mathrm{kDa}$ PEG-maleimide did not change receptor binding affinity. ${ }^{2}$

Based on the in silico studies, E31C and A118C analogs were chosen as PEGylation-positive and -negative models. Further verification was obtained when E31C and A118C analogs showed the same biologic activity, which further 


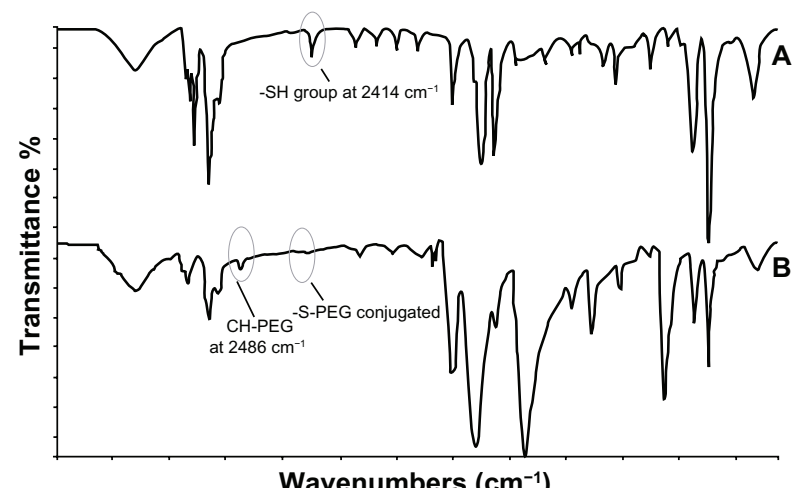

Figure 6 Characterization of the E3IC analog and its PEGylated form. Fourier transform infrared spectra of the E3IC analog before (A) and after (B) PEGylation reaction. A sharp peak at $2414 \mathrm{~cm}^{-1}$ is shown for the sulfhydryl group of free cysteine at position 31. Additionally, the omission of an observed peak at $2414 \mathrm{~cm}^{-1}$ is assumed for E3 IC analog-PEG conjugation. Also, the observation of a new apparent peak at $2486 \mathrm{~cm}^{-1}$ (CH-PEG) is a second confirmation of PEGylated conjugate synthesis.

proves that no structural or functional changes occurred (data not shown). The A118C analog showed a low PEGylation yield in the same conditions, as expected, because of low cysteine accessibility (see Table 3 ). In the case of the E31C analog, FTIR spectra confirmed both the existence of replaced cysteine analog and chemoselective PEGylation. The data also showed an increase in nanosize of EPO and an increase in half-life. Extended terminal half-life due to attachment of $30 \mathrm{kDa} P E G$ is in agreement with findings from previous studies. ${ }^{12,34}$ The attachment of a $30 \mathrm{kDa}$ PEG to the E31C ana$\log$ results in a five-fold increase in half-life, as reported by
Agoram et al. ${ }^{12}$ It has been shown that PEGylation influences protein clearance and plays a crucial role in their elimination from the circulation. ${ }^{6}$ A 10 -fold decrease in clearance of PEGylated E31C analog with $30 \mathrm{kDa}$ PEG is comparable with another study in which a $30 \mathrm{kDa}$ PEG attached to the NM385 variant. ${ }^{12}$ In addition, it is reported that PEGylated proteins' hydrodynamic volumes markedly increased as well. ${ }^{35}$ The present data propound two additional important criteria for nanoEPO half-life or biologic activity enhancement observations: i) increasing the size by PEGylation, as indicated in Figure 7A; and ii) the PEGylation was also found to be helpful in making the protein charge more negative rather than the intact EPO as well. On the other hand, an increase in EPO size (from $3 \mathrm{~nm}$ to $6 \mathrm{~nm}$ ) or a negative charge resulted in a significant increase in biologic characteristics of new nanosize EPO. With this in mind, it has also been shown that the increase or decrease in the protein nanosize or charge by PEGylation may significantly affect protein biologic properties, including half-life, potency, and stability. ${ }^{15,16}$

Previous investigations have elucidated that longeracting products can be created by amine-reactive PEG conjugation. ${ }^{36-40}$ However, as discussed, the PEGylated products consist of multiple PEGylated forms, and varying biologic activities have usually reduced activity due to the conjugation extent of the protein. Many studies have been carried out on PEG conjugation of EPO, but the only marketed form is $\mathrm{CERA}^{\circledR}$, which is attained by conjugation
A
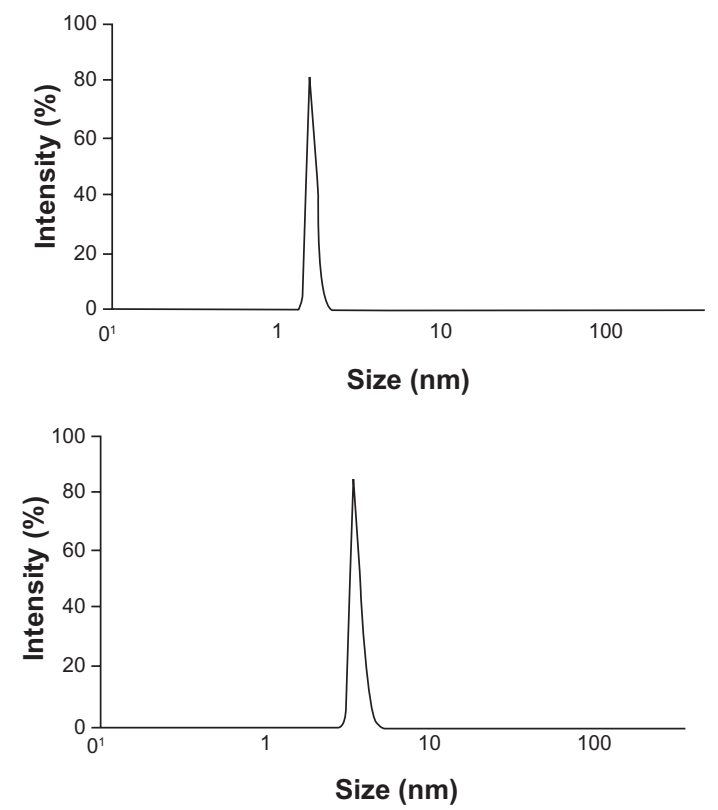

B
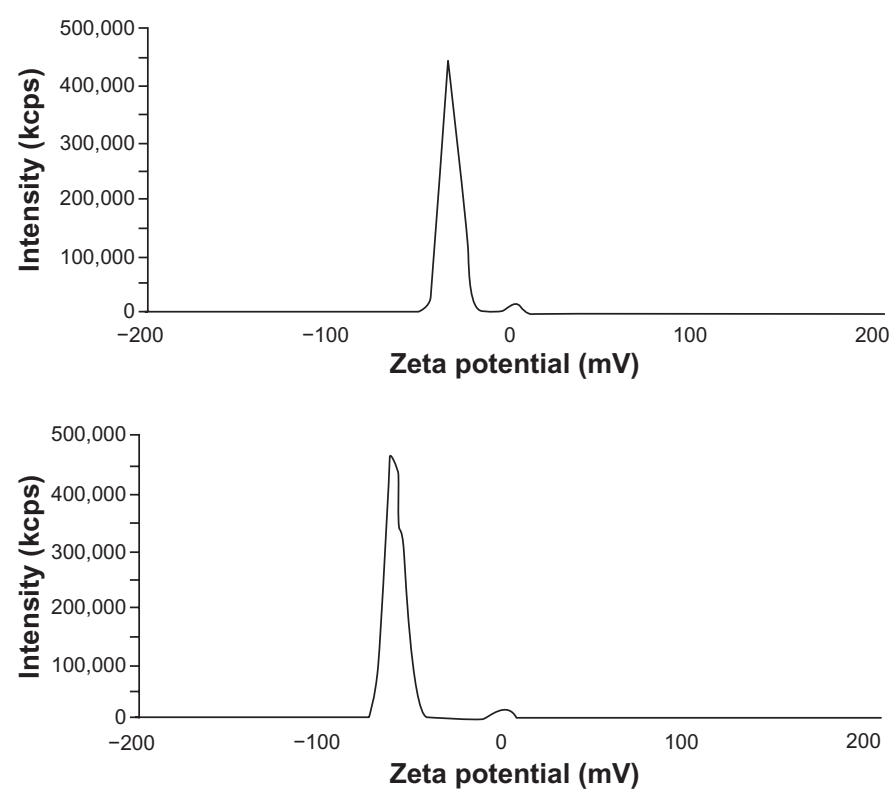

Figure 7 Changes in (a) size and (b) zeta potential of E3IC analog before (up) and after (down) attaching to the PEG-30 kDa. As depicted, the size and negative charge of molecule increased due to the attaching of the polymer. 
Table 4 Pharmacokinetic parameters for the E3IC analog and its PEGylated form after injection of a single dose into the vein tail of rats. As shown, EPO3I-PEG has a lower elimination rate for unmodified protein that leads to a five-fold terminal half-life in plasma

\begin{tabular}{lcc}
\hline $\begin{array}{l}\text { Pharmacokinetic } \\
\text { parameters }\end{array}$ & E3 IC analog & EPO3 I-PEG \\
\hline Elimination rate constant $\left(\mathrm{h}^{-1}\right)$ & $0.096 \pm 0.0 \mathrm{II}$ & $0.019 \pm 0.003$ \\
Terminal half-life $(\mathrm{h})$ & $7.22 \pm 1.3$ & $36.47 \pm 6.5$ \\
$\begin{array}{l}\text { Area under the curve }(0-\infty) \\
\text { (ng.h/mL) }\end{array}$ & $13205.22 \pm 190.7$ & $70238.03 \pm 1 \mathrm{I} 4.4$ \\
Clearance $(\mathrm{mL} / \mathrm{h} / \mathrm{kg})$ & $5.42 \pm 0.82$ & $0.46 \pm 0.04$ \\
\hline
\end{tabular}

Note: The data are represented as mean \pm standard deviation.

of the PEG chain at the N-terminus and lysine residues predominantly at positions 45 or 52. 12,13,41 TGase-mediated PEGylation, provided by Centocor, allows the attaching of one to three PEGs to the protein, but the identity of the modified residue could not be ascertained completely. ${ }^{42}$ Therefore, the major problem associated with PEGylation is due to nonselective conjugation of protein, which leads to heterogeneity or a significant decrease in receptor affinity. Our findings show that when a high-molecular-weight PEG has been conjugated to a protein chemoselectively, then the aforementioned problem does not arise. ${ }^{16}$ As a result, our proposed in silico approach could be used as a promising methodology for rational PEGylation to remove all inconsistencies encountered.

\section{Conclusion}

The nanosize PEGylated E31C-EPO developed in this study shows better pharmacokinetic properties than rhEPO in rats, and further studies will be performed to prove its reliability in the management of anemia. Our results prove that using molecular modeling and MD simulations in rational site selection of cysteine-specific PEGylation is an emerging strategy in developing long-acting molecules. It is possible to use a similar approach to create long-acting forms of other recombinant proteins. Knowledge of factors affecting PEGylation in combination with computational techniques enable us to PEGylate proteins through cysteine residue rationally, without any of the problems that are common in other methods dealing with attaching a high-molecularweight polymer to a protein.

\section{Acknowledgment}

This project was financially supported by the Pasteur Institute of Iran. The computational resource for running molecular dynamic simulations was provided by the National Institute of Genetic Engineering and Biotechnology (NIGEB).
The authors wish to express their deep gratitude to all who provided support during the course of this research.

\section{Disclosure}

The authors report no conflicts of interest in this work.

\section{References}

1. Elliott S, Pham E, Macdougall IC. Erythropoietins: a common mechanism of action. Exp Hematol. 2008;36(12):1573-1584.

2. Long DL, Doherty DH, Eisenberg SP, et al. Design of homogeneous, monopegylated erythropoietin analogs with preserved in vitro bioactivity. Exp Hematol. 2006;34(6):697-704.

3. Jelkmann W. Control of erythropoietin gene expression and its use in medicine. Methods Enzymol. 2007;435:179-197.

4. Wurm FM. Production of recombinant protein therapeutics in cultivated mammalian cells. Nat Biotechnol. 2004;22(11):1393-1398.

5. Testa U. Erythropoietic stimulating agents. Expert Opin Emerg Drugs. 2010;15(1):119-138.

6. Wang Y-J, Chen J, Hao S-J, et al. Efficient preparation and PEGylation of recombinant human non-glycosylated erythropoietin expressed as inclusion body in E. coli. Int J Pharm. 2010;386:156-164.

7. Elliott S, Giffin J, Suggs S, et al. Secretion of glycosylated human erythropoietin from yeast directed by the [alpha]-factor leader region. Gene. 1989;79(1):167-180.

8. Pasut G, Veronese FM. PEGylation for improving the effectiveness of therapeutic biomolecules. Drugs Today. 2009;45(9):687-695.

9. Tillmann HC, Kuhn B, Kränzlin B, et al. Efficacy and immunogenicity of novel erythropoietic agents and conventional rhEPO in rats with renal insufficiency. Kidney Int. 2006;69(1):60-67.

10. Jevševar S, Kunstelj M, Porekar VG. PEGylation of therapeutic proteins. Biotechnol J. 2010;5(1):113-128.

11. Fee CJ, Van Alstine JA. PEG-proteins: reaction engineering and separation issues. Chem Eng Sci. 2006;61(3):924-939.

12. Agoram B, Doshi S, Gegg C, et al. Investigation of the effects of altered receptor binding activity on the clearance of erythropoiesis-stimulating proteins: nonerythropoietin receptor-mediated pathways may play a major role. J Pharm Sci. 2009;98(6):2198-2211.

13. Macdougall IC, Eckardt KU. Novel strategies for stimulating erythropoiesis and potential new treatments for anaemia. Lancet. 2006; 368(9539):947-953.

14. Gaberc-Porekar V, Zore I, Podobnik B, Menart V. Obstacles and pitfalls in the PEGylation of therapeutic proteins. Curr Opin Drug Discov Devel. 2008;11(2):242-250.

15. Chiu K, Agoubi LL, Lee I, et al. Effects of polymer molecular weight on the size, activity, and stability of PEG-functionalized trypsin. Biomacromolecules. 2010;11(12):3688-3692.

16. Maldiney T, Richard C, Seguin J, et al. Effect of core diameter, surface coating, and PEG chain length on the biodistribution of persistent luminescence nanoparticles in mice. ACS Nano. 2011;3:3.

17. Sali A, Blundell TL. Comparative protein modelling by satisfaction of spatial restraints. J Mol Biol. 1993;234(3):779-815.

18. Eramian D, Devos D, Melo F, et al. A composite score for predicting errors in protein structure models. Protein Science. 2006;15: 1653-1666.

19. Lindahl E, Hess B, van der Spoel D. GROMACS 3.0: a package for molecular simulation and trajectory analysis. J Mol Model. 2001;7(8): 306-317.

20. Gans JD, Shalloway D. Qmol: a program for molecular visualization on windows-based PCs. J Mol Graph Model. 2001;19(6):557-579, 609.

21. Delphine CB, David MR, Jan HJ. Very fast prediction and rationalization of pKa values for protein-ligand complexes. Proteins. 2008;73(3): 765-783.

22. Fraczkiewicz R, Braun W. Exact and efficient analytical calculation of the accessible surface areas and their gradients for macromolecules. J Comp Chem. 1998;19:319-333. 
23. Meyer TS, Lamberts BL. Use of Coomassie brilliant blue R250 for the electrophoresis of microgram quantities of parotid saliva proteins on acrylamide-gel strips. Biochim Biophys Acta. 1965;107(1):144-145.

24. Amanlou M, Siadat SD, Alavi A, et al. Gd ${ }^{3+}$-DTPA-DG: novel nanoszied dual anticancer and molecular imaging agent. Int J Nanomedicine. 2011; 6;747-763

25. Roberts MJ, Bentley MD, Harris JM. Chemistry for peptide and protein PEGylation. Adv Drug Deliv Rev. 2002;54(4):459-476.

26. Desjarlais JR, Zalevsky J. Methods for rational PEGylation of proteins. United States Patent and Trademark Office Pre-Grant Publication; 2007.

27. Ibragimova GT, Wade RC. Importance of explicit salt ions for protein stability in molecular dynamics simulation. Biophys $J$. 1998;74(6):2906-2911.

28. Boissel JP, Lee WR, Presnell SR, et al. Erythropoietin structure-function relationships. Mutant proteins that test a model of tertiary structure. J Biol Chem. 1993;268(21):15983-15993.

29. Wen D, Boissel JP, Showers M, et al. Erythropoietin structure-function relationships. Identification of functionally important domains. J Biol Chem. 1994;269(36):22839-22846.

30. Wang YS, Youngster S, Grace M, et al. Structural and biologic characterization of PEGylated recombinant interferon alpha-2b and its therapeutic implications. Adv Drug Deliv Rev. 2002;54(4):547-570.

31. Huaizhi D, Min Z, Yu Z, Chunhua Y. Synthesis and purification of mono-PEGylated insulin. Chem Biol Drug Des. 2007;69(2):132-138.

32. Syed RS, Reid SW, Li C, et al. Efficiency of signalling through cytokine receptors depends critically on receptor orientation. Nature. 1998;395(6701):511-516.

33. Receny MA, Scoble HA, Kim Y. Structural characterization of natural human urinary and recombinant DNA-derived erythropoietin. J Biol Chem. 1987;262(35):17156-17163.

34. Wang YJ, Hao SJ, Liu YD, et al. PEGylation markedly enhances the in vivo potency of recombinant human non-glycosylated erythropoietin: A comparison with glycosylated erythropoietin. $J$ Control Release. 2010;145:306-313.
35. Filpula D, Zhao H. Releasable PEGylation of proteins with customized linkers. Adv Drug Deliv Rev. 2008;60(1):29-49.

36. Clark R, Olson K, Fuh G, et al. Long-acting growth hormones produced by conjugation with polyethylene glycol. J Biol Chem. 1996;271(36):21969-21977.

37. Basu A, Yang K, Wang M, et al. Structure-function engineering of interfferon- $\beta$-1b for improving stability, solubility, potency, immunogenicity, and pharmacokinetic properties by site-selective mono-PEGylation. Bioconjug Chem. 2006;17(3):618-630.

38. Bailon P, Palleroni A, Schaffer CA, et al. Rational design of a potent, long-lasting form of interferon: a $40 \mathrm{kDa}$ branched polyethylene glycolconjugated interferon $\alpha-2 \mathrm{a}$ for the treatment of hepatitis C. Bioconjug Chem. 2001;12(2):195-202.

39. Baker DP, Lin EY, Lin K, et al. N-terminally PEGylated human interferon- $\beta$-1a with improved pharmacokinetic properties and in vivo efficacy in a melanoma angiogenesis model. Bioconjug Chem. 2006;17(1):179-188.

40. Tsutsumi Y, Kihira T, Tsunoda SI, et al. Polyethylene glycol modification of interleukin-6 enhances its thrombopoietic activity. J Control Release. 1995;33(3):447-450.

41. Macdougall IC. CERA (continuous erythropoietin receptor activator): a new erythropoiesis-stimulating agent for the treatment of anemia. Curr Hematol Rep. 2005;4(6):436-440.

42. Pool C, Inventor. Formation of novel erythropoietin conjugates using transglutaminase; 2006.

43. Takeuchi M, Takasaki S, Shimada M, Kobata A. Role of sugar chains in the in vitro biologic activity of human erythropoietin produced in recombinant Chinese hamster ovary cells. J Biol Chem. 1990;265(21):12127-12130.

44. Eisuke T, Gosei K, Masatsugu U, et al. The role of carbohydrate in recombinant human erythropoietin. Eur J Biochem. 1990;188(2): 405-411.

45. Wen D, Boissel JP, Tracy TE, et al. Erythropoietin structure-function relationships: high degree of sequence homology among mammals. Blood. 1993;82(5):1507-1516.
International Journal of Nanomedicine

\section{Publish your work in this journal}

The International Journal of Nanomedicine is an international, peerreviewed journal focusing on the application of nanotechnology in diagnostics, therapeutics, and drug delivery systems throughou the biomedical field. This journal is indexed on PubMed Central,

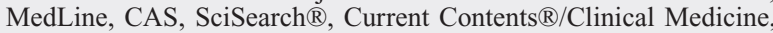

\section{Dovepress}

Journal Citation Reports/Science Edition, EMBase, Scopus and the Elsevier Bibliographic databases. The manuscript management system is completely online and includes a very quick and fair peer-review system, which is all easy to use. Visit http://www.dovepress.com/ testimonials.php to read real quotes from published authors. 